\title{
CONHECIMENTO DE PAIS E CUIDADORES PORTUGUESES SOBRE PRIMEIROS SOCORROS EM ACIDENTES DOMÉSTICOS
}

\author{
KNOWLEDGE OF PORTUGUESE PARENTS \\ AND CARETAKERS ABOUT FIRST AID \\ IN DOMESTIC ACCIDENTS
}

\section{CONOCIMIENTO DE PADRES Y CUIDADORES PORTUGUESES SOBRE PRIMEROS AUXILIOS EN ACCIDENTES DOMÉSTICOS}

\author{
Carolai Conceição dos Santos ${ }^{1}$ \\ Marcia Maria Carneiro Oliveira ${ }^{2}$ \\ Maria Carolina Ortiz Whitaker ${ }^{3}$ \\ Climene Laura de Camargo ${ }^{4}$ \\ Filomena Martins Marcos Raimundo 5 \\ Maria do Carmo Sousa ${ }^{6}$
}

Como citar este artigo: Santos CC, Oliveira MMC, Witaker MCO, Camargo CL, Raimundo FMM, Sousa MC. Conhecimento de pais e cuidadores portugueses sobre primeiros socorros em acidentes domésticos. Rev baiana enferm. 2019;33:e31874.

\begin{abstract}
Objetivo: identificar o nível de conhecimento de pais/cuidadores de crianças sobre primeiros socorros em acidentes domésticos e se existe associação com fatores sociodemográficos. Método: estudo transversal, realizado no Centro Hospitalar de Trás os Montes e Alto Douro em Vila Real, Portugal, durante as consultas pediátricas ambulatoriais, entre maio e junho de 2018. Foram incluídos pais/cuidadores de crianças de 5 a 9 anos e excluídos pais/cuidadores que não tinham nacionalidade portuguesa. A população foi dividida em dois subgrupos, de acordo com o nível de conhecimento: $\leq 19$ acertos (conhecimento fraco/razoável); $\geq 20$ (conhecimento bom/muito bom). Utilizou-se, para a coleta de dados, um formulário contendo 27 questões. Resultados: dentre os 54 participantes, 33 (61,2\%) possuíam conhecimento bom/muito bom; predominou a falta de conhecimento dos cuidados com ferimentos após queda e queimadura. Conclusão: apesar de o conhecimento dos pais/cuidadores ter sido avaliado como bom/muito bom, não houve associação do conhecimento com fatores sociodemográficos.
\end{abstract}

Descritores: Acidentes Domésticos. Crianças. Primeiros Socorros. Conhecimento.

Objective: identifying the level of knowledge of parents/caretakers of children with regards to first aid in domestic accidents, and whether it has any association with sociodemographic factors. Method: cross-sectional study, carried

\footnotetext{
Enfermeira. Pesquisadora Independente. Salvador, Bahia, Brasil. carolaiisantos I2@gmail.com https://orcid.org/0000-000 I-6533-4237

Enfermeira. Doutora em Medicina e Saúde. Professora Adjunta da Universidade Federal da Bahia. Salvador, Bahia, Brasil. https://orcid.org/0000-0003-2294-0872

Enfermeira. Doutora em Ciências da Saúde. Professora Adjunta da Universidade Federal da Bahia. Salvador, Bahia, Brasil. https://orcid.org/0000-0003-0253-383

Enfermeira. Doutora em Saúde Pública. Professora Titular da Universidade Federal da Bahia. Salvador, Bahia, Brasil. https://orcid.org/0000-0002-4880-3916

Enfermeira. Mestre em Educação para Saúde. Professora Adjunta da Universidade de Trás-os-Montes e Alto Douro. Vila Real, Portugal. https://orcid.org/0000-00022060-1204

Enfermeira. Professora da Universidade de Trás-os-Montes e Alto Douro. Vila Real, Portugal. https://orcid.org/0000-0002-72I 5-9307
} 
out in the Hospital Center in Trás os Montes and Alto Douro, in Vila Real, Portugal, during pediatric outpatient consultations from May to June 2018. Parents/caretakers of children from 5 to 9 years of age were included, and parents/caretakers who were not Portuguese were excluded. The population was divided in two subgroups, according to the level of knowledge: $\leq 19$ right answers (little/medium knowledge); $\geq 20$ (good/very good knowledge). A form with 27 questions was used for data collection. Results: among the 54 participants, 33 (61.2\%) had good/very good knowledge; the lack of knowledge about first aid after wounds caused by falls and burns was prevalent. Conclusion: although the knowledge of parents/caretakers was found to be good/very good, there was no significant association of their knowledge with sociodemographic factors.

Descriptors: Accidents, Home. Children. First Aid. Knowledge.

Objetivo: identificar el nivel de conocimiento de padres/cuidadores de niños sobre primeros auxilios en accidentes domésticos y si existe asociación con factores sociodemográficos. Método: estudio transversal, realizado en el Centro Hospitalario Trás os Montes e Alto Douro, en Vila Real, Portugal, durante consultas pediátricas ambulatorias, entre mayo y junio de 2018. Se incluyeron padres/cuidadores de niños de 5 a 9 años y se excluyeron a los padres/cuidadores que no tenían nacionalidad portuguesa. La población se dividió en dos subgrupos, según el nivel de conocimiento: $\leq 19$ aciertos (conocimiento flaco/razonable); $\geq 20$ (buen/muy buen conocimiento). Para recopilación de datos, se utilizó formulario con 27 preguntas. Resultados: entre los 54 participantes, 33 (61,2\%) tenían conocimiento bueno/muy bueno; predominó el desconocimiento sobre el cuidado de las heridas después de caerse y quemarse. Conclusión: aunque el conocimiento de padres/cuidadores se evaluara como bueno/muy bueno, no bubo asociación del conocimiento con factores sociodemográficos.

Descriptores: Accidentes Domésticos. Niños. Primeros Auxilios. Conocimiento.

\section{Introdução}

Os acidentes são as principais causas de morbidade e mortalidade nas crianças, sendo um grave problema de saúde pública ${ }^{(1)}$. De acordo com o Fundo das Nações Unidas para a Infância (UNICEF), milhares de crianças morrem em decorrência de acidentes todos os anos no mundo ou ficam com sequelas permanentes ${ }^{(2)}$. Em Portugal, em 2015, o número total de acidentes domésticos envolvendo crianças na faixa etária entre 5 e 9 anos registrados pelo sistema foi de $2.503^{(1)}$.

A Organização Mundial de Saúde define acidente como qualquer acontecimento, independente da vontade do homem, caracterizado pela libertação súbita de uma força externa ${ }^{(3)}$. Os acidentes domésticos são caracterizados como aqueles registrados nas urgências do Serviço Nacional de Saúde, cuja causa não seja doença, acidente automobilístico, acidente de trabalho ou violência ${ }^{(4)}$. Em Portugal, no período de 2000 a 2013, houve mais de 60.500 internamentos de crianças e jovens relacionados com acidentes ${ }^{(5)}$.

Crianças em idade escolar possuem algumas características próprias do seu processo de crescimento e desenvolvimento que as deixam mais suscetíveis aos acidentes ${ }^{(6)}$. Na faixa etária de 5 a 9 anos, 2.503 crianças portuguesas foram vítimas de acidentes dométicos no período de 2013 a 2015. As quedas, intoxicações e queimaduras estão incluídas entre os acidentes domésticos mais frequentes e que ocorreram nas residências e escolas ${ }^{(1)}$.

Nesse contexto, pode-se afirmar que os cuidados imediatos prestados pelos principais responsáveis são determinantes para minimizar os danos e as sequelas resultantes dos acidentes domésticos em crianças. Portanto, é importante que pais/cuidadores possuam conhecimentos e saibam atuar nos casos de acidente $e^{(7-9)}$.

Os primeiros socorros constituem-se no atendimento inicial e temporário prestado a acidentados e/ou vítimas de doença súbita, com o objetivo de prevenir, alertar ou socorrer, num esforço de preservar a vida, diminuir a incapacidade e minorar o sofrimento ${ }^{(10)}$. Sendo assim, é essencial que aqueles que presenciam ou fazem o primeiro contato estejam capacitados para prestar cuidados adequados e de imediato, no 
local do acidente, para minimizar os riscos de sequelas e aumentar as chances de sobrevida ${ }^{(9)}$.

Estudos apontam que o conhecimento de pais é insuficiente e inadequado para realizar os primeiros socorros em crianças vítimas de acidentes $^{(7-9)}$. Este estudo faz parte dos produtos desenvolvidos em um projeto de Mobilidade Acadêmica, entre a Escola de Enfermagem (UFBA) e a Escola de Enfermagem da Universidade de Trás os Montes, Portugal.

O presente estudo tem por objetivo identificar o nível de conhecimento dos pais/cuidadores de crianças sobre primeiros socorros em acidentes domésticos e se existe associação com fatores sociodemográficos.

\section{Método}

Trata-se de um estudo de corte transversal. A coleta de dados ocorreu no período de maio a junho de 2018, na consulta externa pediátrica do Centro Hospitalar de Trás os Montes e Alto Douro, em Portugual. A população do estudo foi constituída de pais/cuidadores de crianças com idade entre 5 e 9 anos. Foi escolhida essa faixa etária, por ser alvo da consulta externa pediátrica. Foram incluídos pais/cuidadores de crianças de 5 a 9 anos e excluídos pais/cuidadores que não tinham nacionalidade portuguesa.

Após a aprovação do Comité de Ética local (n. 167/2018) foi realizada a aproximação com os pais/cuidadores, por meio de um convite impresso e distribuído individualmente. Nesse momento, eles foram informados sobre os objetivos da pesquisa e foi-lhes solicitado o consentimento formal, por meio da assinatura do Termo de Consentimento Livre e Esclarecido.
Como instrumento de coleta de dados foi elaborado um formulário baseado no manual de primeiros socorros do Instituto Nacional de Emergência Médica de Portugal (INEM) e do Centro Hospitalar de Trás os Montes e Alto Douro (CHTMAD). Esse manual foi utilizado por incluir recomendações importantes, que podem ser aplicadas com crianças de 5 a 9 anos. O formulário elaborado continha 27 questões. Após a análise dos dados obtidos, a população foi dividida em dois subgrupos, de acordo com o nível de conhecimento: $\leq 19$ acertos (conhecimento fraco/razoável); $\geq 20$ (conhecimento bom/muito bom).

Os dados coletados foram processados no Programa Statistic Program for Social Sciences (SPSS) versão 21.0. O teste de normalidade utilizado foi o Kolmogorov-Smirnov. Foram calculadas as frequências absolutas e relativas para as variáveis categóricas. Realizou-se a análise bivariada por meio do Teste Qui-quadrado ( $\chi 2$ ) para conhecer diferenças estatisticamente significativas entre os grupos e Teste $T$ de Student para comparar médias. O nível de significância estatística foi de 5\%.

\section{Resultados}

A população foi composta por 54 indivíduos. Desses, 52 pais e mães (96,3\%) e 2 cuidadores $(3,7 \%)$. O conhecimento foi fraco/razoável em $21(38,8 \%)$ participantes e bom/muito bom em $33(61,2 \%)$ dentre eles. A caracterização da população do estudo e a comparação dos subgrupos de acordo com o nível de conhecimento estão descritos na Tabela 1.

Tabela 1 - Caracterização sociodemográfica da população do estudo e associação dos subgrupos segundo o nível de conhecimento. Trás os Montes e Alto Douro, Portugal - 2018

(continua)

\begin{tabular}{|c|c|c|c|c|}
\hline \multirow[b]{2}{*}{ Variáveis } & \multirow{2}{*}{$\begin{array}{l}\text { População } \\
\text { n=54 (\%) }\end{array}$} & \multicolumn{2}{|c|}{ Conhecimento } & \multirow[b]{2}{*}{ P-valor } \\
\hline & & $\begin{array}{c}\text { Fraco/razoável } \\
\text { n=21 (\%) }\end{array}$ & $\begin{array}{c}\text { Bom/muito bom } \\
\text { n=33 (\%) }\end{array}$ & \\
\hline $\begin{array}{l}\text { Idade (anos) média } \pm \text { desvio } \\
\text { padrão }\end{array}$ & $38 \pm 7$ & $36 \pm 7$ & $39 \pm 7$ & $0,129 *$ \\
\hline \multicolumn{5}{|l|}{ Sexo - n (\%) } \\
\hline Feminino & $38(70,4)$ & $15(71,4)$ & $23(69,7)$ & $0,892^{* * *}$ \\
\hline Masculino & $16(29,6)$ & $6(28,6)$ & $10(30,3)$ & \\
\hline
\end{tabular}


Tabela 1 - Caracterização sociodemográfica da população do estudo e associação dos subgrupos segundo o nível de conhecimento. Trás os Montes e Alto Douro, Portugal - 2018 (conclusão)

\begin{tabular}{|c|c|c|c|c|}
\hline \multirow[b]{2}{*}{ Variáveis } & \multirow{2}{*}{$\begin{array}{l}\text { População } \\
\text { n=54 (\%) }\end{array}$} & \multicolumn{2}{|c|}{ Conhecimento } & \multirow[b]{2}{*}{ P-valor } \\
\hline & & $\begin{array}{c}\text { Fraco/razoável } \\
\text { n=21 (\%) }\end{array}$ & $\begin{array}{c}\text { Bom/muito bom } \\
\text { n=33 (\%) }\end{array}$ & \\
\hline \multicolumn{5}{|l|}{ Escolaridade - n (\%) } \\
\hline Ensino Básico & $13(24,1)$ & $6(28,6)$ & $7(21,2)$ & $0,812^{* *}$ \\
\hline Ensino secundário completo & $22(40,7)$ & $10(47,6)$ & $12(36,4)$ & \\
\hline Ensino secundário incompleto & $6(11,1)$ & $2(9,5)$ & $4(12,1)$ & \\
\hline Ensino superior & $13(24,1)$ & $3(14,3)$ & $10(30,3)$ & \\
\hline \multicolumn{5}{|l|}{ Profissão - n(\%) } \\
\hline Serviços Gerais & $17(31,5)$ & $4(19,0)$ & $13(39,4)$ & $0,183^{* *}$ \\
\hline Forças armadas & $1(1,8)$ & - & $1(3,0)$ & \\
\hline Poder legislativo & $5(9,3)$ & $2(9,5)$ & $3(9,1)$ & \\
\hline Técnicos de nível intermediário & $5(9,3)$ & $4(19,0)$ & $1(3,0)$ & \\
\hline Agricultores & $2(3,7)$ & $2(9,5)$ & - & \\
\hline Industriários & $1(1,8)$ & - & $1(3,0)$ & \\
\hline Operadores de Instalações & $1(1,8)$ & $1(4,8)$ & - & \\
\hline Intelectuais & $13(24,1)$ & $5(23,8)$ & $8(24,2)$ & \\
\hline Não qualificados & $9(16,7)$ & $3(14,3)$ & $6(18,2)$ & \\
\hline
\end{tabular}

Fonte: Elaboração própria.

Notas:

* Teste $t$ de student. ${ }^{* *}$ Qui-quadrado de Pearson.

Sinal convencional utilizado:

- Dado numérico igual a zero não resultante de arredondamento.

Os dados relacionados ao percentual do conhecimento em casos de queda, intoxicação e queimadura, estão descritos nas Tabelas 2, 3 e 4 , respectivamente.

Tabela 2 - Dados relacionados ao conhecimento dos pais/cuidadores com primeiros socorros após queda. Trás os Montes e Alto Douro, Portugal - 2018

(continua)

\begin{tabular}{l|c|c}
\hline \multirow{2}{*}{ Variáveis } & \multicolumn{2}{c}{ Queda } \\
\cline { 2 - 3 } & $\begin{array}{c}\text { Acertos } \\
\text { n (\%) }\end{array}$ & $\begin{array}{c}\text { Erros } \\
\text { n (\%) }\end{array}$ \\
\hline $\begin{array}{l}\text { Quando a criança cai de um nível superior e apresenta edema, hematoma } \\
\text { ou ferida aberta, proteger a zona com compressas limpas, não mobilizar e } \\
\text { chamar o 112. }\end{array}$ & $413(24,1)$ \\
$\begin{array}{l}\text { Em caso de queda, manter a criança sob observação e não oferecer } \\
\text { qualquer alimento até que a criança volte à sua atividade e }\end{array}$ & $48(88,9)$ & $6(11,1)$ \\
comportamento habituais. & \\
$\begin{array}{l}\text { Em queda de altura semelhante ao da criança, quando esta não apresenta } \\
\text { alteração do estado de consciência, mas apresenta ferida aberta pouco } \\
\text { extensa e pouco profunda, devem-se observar as caraterísticas da ferida, } \\
\text { proceder à sua limpeza com água tépida e fazer penso compressivo. }\end{array}$ \\
$\begin{array}{l}\text { Após uma queda, sempre que a criança apresentar alteração do estado } \\
\text { de consciência ou convulsão, deve-se mantê-la calma, não mobilizar e } \\
\text { chamar 112. }\end{array}$ \\
\hline
\end{tabular}


Tabela 2 - Dados relacionados ao conhecimento dos pais/cuidadores com primeiros socorros após queda. Trás os Montes e Alto Douro, Portugal - 2018

\begin{tabular}{|c|c|c|}
\hline \multirow[b]{2}{*}{ Variáveis } & \multicolumn{2}{|c|}{ Queda } \\
\hline & $\begin{array}{c}\text { Acertos } \\
\text { n (\%) }\end{array}$ & $\begin{array}{l}\text { Erros } \\
\text { n (\%) }\end{array}$ \\
\hline $\begin{array}{l}\text { Em caso de queda, observar com atenção o aparecimento de náuseas, } \\
\text { vómitos e sonolência na criança, e se esta apresentar qualquer desses } \\
\text { sintomas deve-se recorrer ao serviço de urgência ou ligar o } 112 .\end{array}$ & $54(100,0)$ & - \\
\hline $\begin{array}{l}\text { Em queda de altura semelhante ao da criança, quando esta não apresenta } \\
\text { alteração do estado de consciência e apresenta edema ou hematoma } \\
\text { aplicar compressas frias na região, fazendo leve pressão. }\end{array}$ & $14(25,9)$ & $40(74,1)$ \\
\hline
\end{tabular}

Tabela 3 - Dados relacionados ao conhecimento dos pais/cuidadores com primeiros socorros após intoxicação. Trás os Montes e Alto Douro, Portugal - 2018

\begin{tabular}{l|c|c}
\hline \multirow{2}{*}{ Variáveis } & \multicolumn{2}{c}{ Intoxicação } \\
\cline { 2 - 3 } & $\begin{array}{c}\text { Acertos } \\
\text { n (\%) }\end{array}$ & $\begin{array}{c}\text { Erros } \\
\text { n (\%) }\end{array}$ \\
\hline $\begin{array}{l}\text { Quando se suspeita de que a criança ingeriu alguma substância tóxica, } \\
\text { deve-se sempre perguntar o que foi que ela ingeriu e procurar encontrar } \\
\text { a embalagem do produto. }\end{array}$ & $51(94,4)$ & $3(5,6)$ \\
$\begin{array}{l}\text { Sempre que a criança ingerir alguma susbtância tóxica, a primeira } \\
\text { medida é manter a calma... }\end{array}$ & $54(100,0)$ & - \\
$\begin{array}{l}\text { Em caso de intoxicação por ingestão de álcool, e apenas neste caso, } \\
\text { deve-se dar uma bebida açucarada à criança. }\end{array}$ & $34(63,0)$ & $20(37,0)$ \\
$\begin{array}{l}\text { Sempre que a criança ingerir substância tóxica e apresenta restos da } \\
\text { mesma na boca, deve-se dar água para bochechar e jogar fora. }\end{array}$ & $43(79,6)$ & $11(20,4)$ \\
$\begin{array}{l}\text { Sempre que a criança ingere um tóxico e se for conhecida a hora da } \\
\text { ingestão, deve-se provocar o vômito. }\end{array}$ & $19(35,2)$ & $35(64,8)$ \\
$\begin{array}{l}\text { Quando se recorre ao serviço de urgência com uma criança que ingeriu } \\
\text { substância tóxica, deve-se levar sempre a embalagem do produto ingerido. }\end{array}$ & $52(96,3)$ & $2(3,7)$ \\
$\begin{array}{l}\text { Sempre que a criança ingere uma substância tóxica, deve-se dar água } \\
\text { para beber. }\end{array}$ & $41(75,9)$ & $13(24,1)$ \\
$\begin{array}{l}\text { Quando uma criança fez inalação de monóxido de carbono, deve-se } \\
\text { oferecer sempre leite. }\end{array}$ & $32(59,3)$ & $22(40,7)$ \\
$\begin{array}{l}\text { Sempre que um produto tóxico entra em contato com a pele de uma } \\
\text { criança, deve-se, de imediato, dar banho na criança. }\end{array}$ & $18(33,3)$ & $36(66,7)$ \\
$\begin{array}{l}\text { Quando um produto tóxico entra em contato com os olhos da criança, } \\
\text { deve-se virar a cabeça para o lado afetado, lavar com água corrente } \\
\text { durante 15 minutos, mantendo as pálpebras afastadas, tendo cuidado } \\
\text { para que o produto não escorra para outras partes do corpo. }\end{array}$ & $51(94,4)$ & $3(5,6)$ \\
\hline
\end{tabular}

Fonte: Elaboração própria.

Nota: Sinal convencional utilizado:

- Dado numérico igual a zero não resultante de arredondamento. 
Tabela 4 - Dados relacionados ao conhecimento dos pais/cuidadores com primeiros socorros após queimadura. Trás os Montes e Alto Douro, Portugal - 2018

\begin{tabular}{l|c|c}
\hline \multirow{2}{*}{ Variáveis } & \multicolumn{2}{c}{ Queimadura } \\
\cline { 2 - 3 } & $\begin{array}{c}\text { Acertos } \\
\text { n (\%) }\end{array}$ & $\begin{array}{c}\text { Erros } \\
\text { n (\%) }\end{array}$ \\
\hline $\begin{array}{l}\text { Quando uma criança sofre uma queimadura é importante saber qual foi } \\
\text { O agente responsável. }\end{array}$ & $43(79,6)$ & $11(20,4)$ \\
Quando uma criança sofre uma queimadura deve-se recorrer sempre ao & $45(83,3)$ & $9(16,7)$ \\
centro de saúde para uma avaliação correta. & & $7(13,0)$ \\
Quando uma criança sofrer uma queimadura, deve-se lavar & $47,0)$ \\
imediatamente o local com água fria corrente, durante 5 minutos, para \\
diminuir a dor e proteger a área com uma compressa limpa e úmida. \\
$\begin{array}{l}\text { Quando uma criança sofreu uma queimadura, nunca se deve colocar } \\
\text { pomadas, pasta de dentes, manteiga ou outros. }\end{array}$ \\
$\begin{array}{l}\text { Quando uma criança que sofreu uma queimadura apresenta flictenas } \\
\text { (bolhas) na pele, vocês devem sempre rebentar para o líquido poder sair } \\
\text { e proteger a zona com compressa. }\end{array}$ \\
\hline
\end{tabular}

Fonte: Elaboração própria.

\section{Discussão}

Neste estudo, identificou-se que o conhecimento dos pais/cuidadores sobre primeiros socorros relacionados à queda, intoxicação e queimaduras em crianças e 5 a 9 anos em Portugal foi considerado bom/muito bom na maioria das variáveis analisadas. Não houve diferença estatisticamente significativa relacionada ao conhecimento com a caracterização sociodemográfica, o que pode estar relacionado com o pequeno tamanho da população.

Quanto ao sexo, os homens encontravam-se em minoria na população total, porém, quando analisado de acordo com o conhecimento bom/muito bom, eles apresentavam maior escore de conhecimento quando comparados às mulheres. Como ocorre na maioria dos países do mundo, na cultura lusitana, a mulher é também considerada como a principal responsável no cuidado do filho (ou principal cuidadora), porém, neste estudo, os homens destacaram-se nas respostas assertivas sobre situações de primeiros socorros com seus filhos ${ }^{(11-13)}$.

O cenário tradicional de mães como cuidadoras vem sofrendo modificações com o passar dos anos, devido à ampliação da mulher no mercado de trabalho e sua atuação como provedora financeira da família. Esta realidade tem contribuído significativamete para que um número cada vez maior de homens assuma papéis que eram destinados exclusivamente ao sexo feminino.

Estudos apontam o aumento da presença masculina em várias situaçõs de cuidado com os filhos, inclusive em casos de adoecimento desses. Isto é, na contemporaneidade, o modelo da paternidade participativa tem sido adotado pelos casais frequentemente, o que subentende o cuidado e o envolvimento constante no cotidiano dos filhos, nos domínios da alimentação, higiene, lazer e educação ${ }^{(12,14)}$.

Outro dado que chamou a atenção foi o grau de escolaridade dos pais/cuidadores, pois a maioria não possuía nivel superior. Estudos identificaram menor nível de escolaridade favorável à ocorrência de acidentes. Isso pode ser devido ao fato de a população do estudo residir em cidade pequena no interior do país, onde há maior facilidade para completar os estudos ${ }^{(13,15)}$.

Quando se analisaram os conhecimentos de primeiros socorros relacionados às quedas, observou-se um percentual de pais/cuidadores que possuíam pouco conhecimento sobre cuidados em casos de lesões na pele (ferimentos e hematomas) após uma queda, importância da 
imobilização e chamada de um socorro. Algumas vezes, pode ocorrer um trauma que não lese de imediato as fibras nervosas da medula, porém a lesão pode surgir posteriormente, em consequência da manipulação inadequada da coluna na cena do acidente ou durante o transporte. Daí a importância do correto atendimento no local ${ }^{(16)}$. Estudo aponta que os leigos possuem conhecimentos incompletos ou incorretos sobre atendimento às vítimas desacordadas ${ }^{(17)}$.

Nas intoxicações, observou-se que muitos pais/cuidadores entendiam que deviam provocar o vômito nos casos de ingestão de qualquer substância tóxica, oferecer leite para ingerir e dar banho após contato do produto tóxico com a pele, no entanto, essas atuações não são sempre as recomendadas. Manual de primeiros socorros do Centro Hospitalar de Trás os Montes e Alto Douro orienta a não provocar o vômito e não oferecer líquidos à vítima após ingestão de qualquer tóxico, pois pode agravar o estado clínico em caso de substâncias corrosivas. O banho não é recomendado. A prioridade é lavar imediatamente o local em água corrente, por pelo menos vinte minutos ${ }^{(16)}$.

Nos casos de queimaduras, observou-se que muitos pais/cuidadores usariam pomadas e cremes de cobertura nas lesões, assim como rompimentos das flictenas. Essas ações aumentam as chances de infecções no local. Estudo sobre o nível de conhecimento dos pais sobre os primeiros socorros das queimaduras efetuado em Portugal destacou que cremes, pomadas ou qualquer outra substância não devem ser utilizados, uma vez que podem interferir na avaliação clínica da lesão. Um estudo também constatou que o nível de conhecimento dos pais sobre as medidas de abordagem inicial em caso de queimadura é insuficiente ${ }^{(8)}$, o que não foi constatado na população do presente estudo. Existe, porém, a necessidade de orientar os pais sobre primeiros socorros, para promover ações preventivas e educativas ${ }^{(13)}$.

O estudo apresenta limitações em relação ao tamanho pequeno da população e ao curto período de coleta de dados, que impedem a generalização dos resultados. Embora este estudo permita identificar o conhecimento dos pais sobre os primeiros socorros prestados às crianças, ainda existem lacunas nesses conhecimentos. Sugerem-se estudos controlados, que possam identificar as repercussões físicas e psicológicas, quando os primeiros socorros são realizados de forma inadequada, e os pais não conseguem salvar a vida das crianças, bem como que avaliem a demanda e os custos para os serviços de saúde.

\section{Conclusão}

A presente investigação permite concluir, que a maioria dos pais/cuidadores de crianças residentes em Trás aos Montes e Alto Douro em Portugal possui conhecimento bom/muito bom sobre os primeiros socorros em caso de acidentes dométicos: queda, intoxicação e queimadura. Apesar de não haver associação do conhecimento com fatores sociodemográficos, observou-se que existe deficiência do conhecimento sobre a importância da imobilização após o trauma e nos casos de intoxicação. Considerando que muitos adotavam práticas desaconselháveis em relação à ingestão de substâncias tóxicas, que podem acarretar várias complicações, é necessário intensificar ações de educação em saúde, principalmente relacionadas com essas temáticas.

Há evidências da necessidade de criação de um programa de educação em saúde para pais e cuidadores sobre primeiros socorros em idade pediátrica, a ser promovido em instituições de saúde e escolas. Profissionais da saúde, como enfermeiras, pediatras e outros membros da equipe multiprofissional, têm um papel importante na prevenção e orientação sobre primeiros socorros em situação de acidentes domésticos, e podem contribuir para reduzir a morbimortalidade infantil.

\section{Colaborações:}

1 - concepção, projeto, análise e interpretação dos dados: Carolai Conceição dos Santos, Márcia Maria Carneiro Oliveira, Maria Carolina Ortiz Whitaker e Climene Laura de Camargo;

2 - redação do artigo e revisão crítica relevante do conteúdo intelectual: Carolai 
Conceição dos Santos, Márcia Maria Carneiro Oliveira, Maria Carolina Ortiz Whitaker, Climene Laura de Camargo, Filomena Martins Marcos Raimundo e Maria do Carmo Sousa;

3 - aprovação final da versão a ser publicada: Carolai Conceição dos Santos, Márcia Maria Carneiro Oliveira, Maria Carolina Ortiz Whitaker, Climene Laura de Camargo e Filomena Martins Marcos Raimundo.

\section{Referências}

1. Alves T, Rodrigues E, Neto M, Mexia R, Dias CM. Acidentes domésticos e de lazer: resultados do sistema de vigilância EVITA apurados para o período 2013-2015. Bol Epidemiol. Inst Nac Saúde [Internet]. 2017 [cited 2016 Jun 29];(10):43-6. Available from: http:// repositorio.insa.pt/bitstream/10400.18/4718/1/ Boletim_Epidemiologico_Observacoes_N18_2017_ artigo10.pdf

2. Romero HSP, Rezende EM, Martins EF. Mortalidade por causas externas em crianças de um a nove anos. REME - Rev Min Enferm. 2016;20:e958. DOI: $10.5935 / 1415-2762.20160027$

3. Ramos TMCF. Prevenção de acidentes domésticos na criança: comportamento parental [Internet]. [dissertação]. Viseu: Instituto Politécnico de Viseu; 2017 [cited 2017 Jun 10]. Available from: http://repositorio.ipv.pt/bitstream/10400.19/ 4501/1/TeresaMariaFernandesRamos\%20DM.pdf

4. Contreiras T, Rodrigues E, Nunes B. Adelia Acidentes Domésticos e de Lazer: Informação adequada. Relatório 2006-2008. Lisboa: Inst Nac Saúde [Internet]. 2010 Dez [cited 2018 Jun 21]: 1-72. Available from: http://repositorio.insa.pt/ bitstream/10400.18/743/1/Relat\%C3\%B3rio\%20 ADELIA\%202006-2008_18_4_2011_final.pdf

5. Costa IP, Nascimento S, Anjos S. Quedas em crianças e jovens: um estudo retrospetivo 2000-2013. ASPSI - Assoc Prom Segurança Infantil [Internet]. 2014 nov (cited 2018 May 18]: 1-15. Available from: http://apsi.org.pt/images/ Documentos/EstudodasQuedas20002013_ Resumo.pdf

6. Hockenberry MJ, Wilson D. Enfermagem da criança e do adolescente. 9a ed. Rio de Janeiro: Lusociência; 2014.

7. Antoniolli L, Bazzan JS, Rosso LH, Amestoy SC, Echevarría-Guanilo ME. Conhecimento da população sobre os primeiros socorros frente à ocorrência de queimaduras: uma revisão integrativa. Rev bras queimaduras [Internet]. 2014 [cited 2017 Jun 5];13(4):251-9. Available from: http://lildbi.bvs. br/lildbi/docsonline/get.php?id=211

8. Barroso F, Lachado A, Melo AR, Pinto F. O que sabem os pais sobre o tratamento de queimaduras. Acta Pediátr Port 2018;49:33-41. DOI: 10.21069/ APP.2018.11947

9. Machado MAS, Ribeiro CS, Souza LR, Costa AL, Filócomo FRF. O conhecimento de professores do ensino fundamental sobre primeiros socorros que devem ser prestados a alunos em ambiente escolar [Internet]. Trabalho apresentado no XI Encontro Latino-Americano de Iniciação Científica, XI Encontro Latino-Americano de Pós-Graduação e V Encontro Latino-Americano de Iniciação Científica Júnior. São José dos Campos: Univap Virtual; 2011. p. 1-6. [cited 2017 Jun 25]. Available from: http://www.inicepg.univap.br/cd/INIC_2011/ anais/arquivos/0274_0776_01.pdf

10. Reis I. Manual de Primeiros Socorros: Situações de urgência nas Escolas, Jardins de Infância e Campos de Férias [Internet]. 3a ed rev. Portugual: Ministério da Educação; 2010 [cited 2018 Jun 8]. Available from: http://www.dge.mec.pt/sites/ default/files/Esaude/primeirossocorros.pdf

11. Martins CA, Abreu WJCP, Figueiredo MCAB. Tornar-se pai e mãe: um papel socialmente construído. Rev Enferm Ref [Internet]. 2014 [cited 2018 Jul 21];serIV(2):121-31. Available from: http://www.scielo.mec.pt/pdf/ref/vserIVn2/ serIVn2a13.pdf

12. Sutter C, Bucher-Maluschke JSNF. Pais que cuidam dos filhos: a vivência masculina na paternidade participativa. Psico [Internet]. 2008 [cited 2018 Jul 21];39:74-82. Available from: http://revistaseletronicas.pucrs.br/ojs/index.php/ revistapsico/article/viewFile/1488/2799

13. Durães MRP, Toriyama ÁTM, Maia LFS. O conhecimento dos pais sobre como proceder diante de acidentes domésticos. Rev Recien [Internet]. 2012 [cited 2018 Jul 10];2(6):5-15. Available from: https://www.recien.com.br/index. $\mathrm{php} /$ Recien/article/view/41/79

14. Barros PN, Andrade AW, Andrade BLAC, Santos AJ, Bittencourt PASL, Fontão ZMM, et al. Care provided by the father to the child with cancer under the influence of masculinities: qualitative meta-synthesis. Rev Bras Enferm [Internet]. 2018 [cited 2018 Jun 20];71(1):197-206. Available 
Carolai Conceição dos Santos, Marcia Maria Carneiro Oliveira, Maria Carolina Ortiz Whitaker, Climene Laura de Camargo, Filomena Martins Marcos Raimundo, Maria do Carmo Sousa

from: http://www.scielo.br/scielo.php?script=sci_ arttext\&pid=S0034-71672018000100185

15. Ramos AL, Nunes L. Passo a passo caminhamos para o sucesso: a problemática dos acidentes em ambiente doméstico/familiar nas crianças. Rev Percursos [Internet]. 2012 Out-Dez [cited 2018 Aug 10];(26):41-8. Available from: https://comum. rcaap.pt/bitstream/10400.26/9252/1/Revista\%20 Percursos\%20n26_A\%20problem\%C3\%A1tica\%20 dos $\% 20$ acidentes $\% 20$ em $\% 20$ a mbiente $\% 20$ dom\%C3\%A9stico\%20e\%20familiar\%20nas\%20 crian\%C3\%A7as.pdf

16. Françoso LA, Malvestio MA. Manual de prevenção de acidentes e primeiros socorros nas Escolas
[Internet]. São Paulo: Secretaria Municipal de Saúde; 2007 [cited 2018 Jun 28]. Available from: https://www.amavi.org.br/arquivo/colegiados/ codime/2016/Primeiros_Socorros_Manual_Prev_ Acid_Escolas.pdf

17. Cabral EV, Oliveira MFA. Primeiros socorros na escola: conhecimento dos professores. Ensino, Saúde Ambiente [Internet]. 2017 abr [cited 2018 Jul 21];10(1):175-86. Available from: http://periodicos.uff. br/ensinosaudeambiente/article/view/21255/12727

Recebido: 23 de maio de 2019 Aprovado: 23 de setembro de 2019

Publicado: 16 de dezembro de 2019

A Revista Baiana de Enfermagem utiliza a Licença Creative Commons - Atribuição-NãoComercial 4.0 Internacional. https://creativecommons.org/licenses/by-nc/4.0/

Este artigo é de acesso aberto distribuído sob os termos da Licença Creative Commons (CC BY-NC). Esta licença permite que outros remixem, adaptem e criem a partir do seu trabalho para fins não comerciais. Embora os novos trabalhos tenham de lhe atribuir o devido crédito e não possam ser usados para fins comerciais, os usuários não têm de licenciar esses trabalhos derivados sob os mesmos termos. 